\title{
Microscale IR thermography: A novel instrument for IR spectroscopic thermal imaging
}

\author{
J. Morikawa ${ }^{1}$, M. Ryu ${ }^{1}$, M.Romano ${ }^{2}$, C. Pradere ${ }^{2}$, and J .C. Batsale ${ }^{2}$ \\ 1 Tokyo Institute of Technology, Department of Organic and Polymeric Materials, Tokyo, \\ Japan \\ 2 I2M, Département TREFLE, UMR CNRS 5295 - site ENSAM Esplanade des Arts et \\ Métiers, 33405 Talence Cedex, France
}

We have been developing the instruments for microscale IR thermography with original optics' designs and a signal superimposed system, which has been applied to (1) pseudo acceleration of temporal resolution of periodic thermal events, (2) pixel by pixel emissivity-temperature conversion, (3) micro-scale flying spot method. A novel portable instrument design for microscale IR thermography improves the differential temperature and temporal resolution of images that can be obtained even with uncooled detectors.

In this study, we describe the new instrumentation of infrared spectroscopic thermal imaging, which enables simultaneous measurements of IR spectra and thermal imaging in microscale spatial resolution. The method can be applied even to dynamic thermal phenomena such as thermodynamic phase transitions. The precise analysis of latent heat evolvement and IR spectrum of organic molecular crystals will be presented.

Keywords: microscale IR thermography, spectroscopic thermal imaging, phase transition 